\title{
BMJ Open Public provision of emergency obstetric care: a case study in two districts of Pakistan
}

\author{
Peter Brückmann, ${ }^{1}$ Ashfa Hashmi, ${ }^{2}$ Marina Kuch, ${ }^{3}$ Jana Kuhnt, ${ }^{4}$ Ida Monfared, ${ }^{5}$ \\ Sebastian Vollmer ${ }^{\circ}$
}

To cite: Brückmann $P$, Hashmi A, Kuch M, et al. Public provision of emergency obstetric care: a case study in two districts of Pakistan. BMJ Open 2019;9:e027187. doi:10.1136/ bmjopen-2018-027187

- Prepublication history for this paper is available online. To view these files, please visit the journal online (http://dx.doi org/10.1136/bmjopen-2018027187).

Received 7 November 2018 Revised 16 March 2019 Accepted 18 April 2019

Check for updates

(C) Author(s) (or their employer(s)) 2019. Re-use permitted under CC BY-NC. No commercial re-use. See rights and permissions. Published by BMJ.

${ }^{1}$ Research and Impact Assessment Division, International Fund for Agricultural Development, Rome Italy

${ }^{2}$ WHO Pakistan Office, Islamabad, Pakistan

${ }^{3} \mathrm{AFC}$ Agriculture and Finance Consultants, Bonn, Germany

${ }^{4}$ German Development Institute, Bonn, Germany

${ }^{5}$ Centre for Modern Indian

Studies \& Department of

Economics, University of

Goettingen, Göttingen, Germany

Correspondence to

Professor Sebastian Vollmer; svollmer@uni-goettingen.de

\section{ABSTRACT}

Objectives Pakistan is one out of five countries where together half of the global neonatal deaths occur. As the provision of services and facilities is one of the key elements vital to reducing this rate as well as the maternal mortality rate, this study investigates the status of the delivery of essential obstetric care provided by the public health sector in two districts in Khyber Pakhtunkhwa in 2015 aiming to highlight areas where critical improvements are needed.

Setting We analysed data from a survey of 22 primary and secondary healthcare facilities as well as 85 community midwives (CMWs) in Haripur and Nowshera districts.

Participants Using a structured questionnaire we evaluated the performance of emergency obstetric care (EmOC) signal functions and patient statistics in public health facilities. Also, 102 CMWs were interviewed about working hours, basic and specialised delivery service provision, referral system and patient statistics.

Primary outcome measures We investigate the public provision of emergency obstetric care using seven key medical services identified by the United Nations (UN).

Results Deliveries by public health cadres account for about $30 \%$ of the total number of births in these districts. According to the UN benchmark, only a small fraction of basic EmOC (2/18) and half of the comprehensive EmOC (2/4) facilities of the recommended minimum number were available to the population in both districts. Only a minority of health facilities and CMWs carry out several signal functions. Only $8 \%$ of the total births in one of the study districts are performed in public EmOC health facilities. Conclusions Both districts show a significant shortage of available public EmOC service provisions. Development priorities need to be realigned to improve the availability, accessibility and quality of EmOC service provisions by the public health sector alongside with existing activities to increase institutional births.

\section{BACKGROUND}

Low-income and middle-income countries (LMICs) continue to struggle to meet the target reductions of maternal and child mortality. ${ }^{1}{ }^{2}$ In Pakistan, estimates in 2015 approximate a maternal mortality rate of 178 women per 100000 live births and a neonatal mortality rate of 46 neonates per 1000 live

\section{Strengths and limitations of this study}

- This study presents primary data from a rural region in Pakistan for which very little is known about the quality of obstetric care.

- Through assessing key service functions identified by the United Nations, this study provides an example where public health facilities fail to meet the requirements for basic emergency obstetric care.

- It adds to the body of evidence needed to improve the quality of neonatal care in low-income settings.

- This is a cross-sectional study using descriptive method thus carries associated limitations of such studies.

It is a case study with limited external validity.

births. A particular challenge is Pakistan's high rate of neonatal mortality in comparison to its neighbouring countries. ${ }^{134}$

In an effort to address the high burden of neonatal and maternal mortality in LMICs, WHO has emphasised the need for a transition from home births to institutional. ${ }^{56}$ This strategy is also acknowledged by the Government of Pakistan, which realigned development priorities to strengthen and expand the institutional service delivery system. ${ }^{7}$ There is also literature questioning the effectiveness of this approach, as the capability of many public health facilities to provide safe births cannot be guaranteed in many LMIC settings. ${ }^{8} 9$ The expansion of institutional births can only be successful if the public health system has the capacity and quality to ensure safe births. Seven key medical services have been identified by the United Nations (UN), which form the basic emergency obstetric care (BEmOC) services. $^{10}$ They comprise the administration of parenteral antibiotics, uterotonic drugs, parenteral anticonvulsants, the manual removal of placenta, the removal of retained products of conception, assisted vaginal delivery and neonatal resuscitation. In addition to these BEmOC 
services, healthcare institutions are regarded as comprehensive EmOC (CEmOC) providers if blood transfusions and caesarean sections are offered. ${ }^{10}$ Providing these services to every mother and child is considered of overarching importance to reduce the maternal and neonatal mortality and morbidity burden. ${ }^{11-15}$

The existing literature for Pakistan highlights the need to improve the availability, accessibility and quality of EmOC services in the public sector. ${ }^{16}$ Several programmes were introduced to address this issue. As such, in 2006, the Ministry of Health of the Government of Pakistan established a national Maternal, Neonatal \& Child Health Programme $(\mathrm{MNCH})$, which emphasised training and deploying community midwives (CMW) ${ }^{17}$ CMWs represent a cadre of non-facility-based skilled birth attendants who perform births either at the midwifery or patient homes to increase skilled birth attendance in underserved communities. They act as a first level of contact for women in the community and are supported by successive levels of referral facilities such as basic health units (BHU) or civil hospitals, however, they are not expected to conduct births at these facilities. Through this and some other similar programmes attempts were made to decentralise the healthcare infrastructure and strengthen and improve the provision of EmOC at the national level, however, the degree of success of integration of $\mathrm{MNCH}$ at the district level remained less known. ${ }^{18}$

Focusing on two rural districts, Nowshera and Haripur in Khyber Pakhtunkhwa (KP), one of the four main provinces in Pakistan, this study investigates the status of the successful delivery of EmOC as well as services provided by CMWs. While similar studies were carried out in other provinces (eg, in Punjab and North West Frontier Province $^{1920}$ and $\operatorname{Sindh}^{21}$ ), evidence from districts in KP to the date of the present study were scarce. By gathering knowledge from these rural areas, this study addresses the fact that successful implementation of programmes such as $\mathrm{MNCH}$ depends on having an assessment of the status of the existing services without which efficient and effective improvements cannot be made. This study presents the results collected during January 2016 that is almost a decade after the introduction of $\mathrm{MNCH}$ and by when the goals of Millennium Development Goals 4 and 5 were expected to be achieved.

\section{METHODS}

\section{Study setting}

According to Pakistan Social and Living Standards Measurement Survey 2013-14, among provinces, KP had the slowest rate of increase in the number of women in rural areas who received a postnatal consultation within 6 months after birth with only two percentage point improvement ${ }^{22}$ between 2007 and 2014, falling behind other provinces noticeably. During this period, in KP there has been a slow change in the type of place of giving birth shifting from home to government hospitals/rural health centres (RHC)/BHU and private facilities where in 2007 in rural areas $71 \%, 9 \%$ and $19 \%$ of cases were in home, for example, hospitals, and private clinics changing to $52 \%, 15 \%$ and $32 \%$ in 2014 , respectively.

Within province, Haripur and Nowshera are two districts where in comparison with urban areas, rural regions have particularly less access to any type of health facilities yet in 2014-15. In Nowshera, $62 \%$ of prenatal consultations took place in government hospitals vs $31 \%$ taking place in private clinics (this rate is unknown for Haripur). ${ }^{23}$ Haripur has an estimated population of 985000 and for Nowshera that is 1.24 million. Based on the national crude birth rate of 29.2 per 1000 population, ${ }^{24}$ the estimated number of live births for Haripur and Nowshera is 28760 and 36200 , respectively.

Primary and secondary level facilities in Haripur and Nowshera offer public obstetric care. Primary healthcare institutions comprised BHUs, RHCs and maternal and child health centres (MCHC). Civil hospitals and District Headquarter Hospitals (DHQH) are secondary level facilities that provide specialised care in larger towns and district capitals. Tertiary care facilities are not available in both districts. In cases of severe complications, health providers in Haripur and Nowshera refer to tertiary healthcare hospitals in the neighbouring districts, namely hospitals in Peshawar and Mardan for pregnancy complications in Nowshera and hospitals in the district of Abottabad for Haripur. CMWs in both districts provide antenatal, intrapartum, delivery, postnatal and newborn care in their communities.

\section{Study sample and survey questionnaires}

A list of public health facilities and trained CMWs in the two districts was obtained from the provincial government. The initial sample covered all 103 public health facilities in these districts: 70 BHUs, 13 RHCs, 8 MCHCs, 10 civil hospitals and 2 DHQHs. Health facilities with no or less than an average of three births per month in the past 6 months were excluded from the survey. Out of the 70 BHUs in Haripur, only $7(10 \%)$ reported births in the previous 6 months. Similar findings are noted for the MCHCs, with only one facility out of eight providing birth services. A questionnaire covering various aspects including basic and specialised birth service provision, monitoring and communication in the team, incoming and outgoing referrals and resource and drug assessment was carried out in 22 health facilities in total, with 14 health facilities in Haripur and 8 in Nowshera (figure 1). The survey was administered throughout January 2016. In total, 12 enumerators with a medical background in obstetric care were trained over a 7-day period to conduct the survey.

Within the two districts, 32 and 53 CMWs were interviewed in Haripur and Nowshera, respectively, making a total of 102 interviews providing a fair representative of both areas (the remaining CMWs were either unavailable or did not allow us to visit them for security reasons). Through this questionnaire, CMWs were asked about basic characteristics of the CMW and her catchment area, 


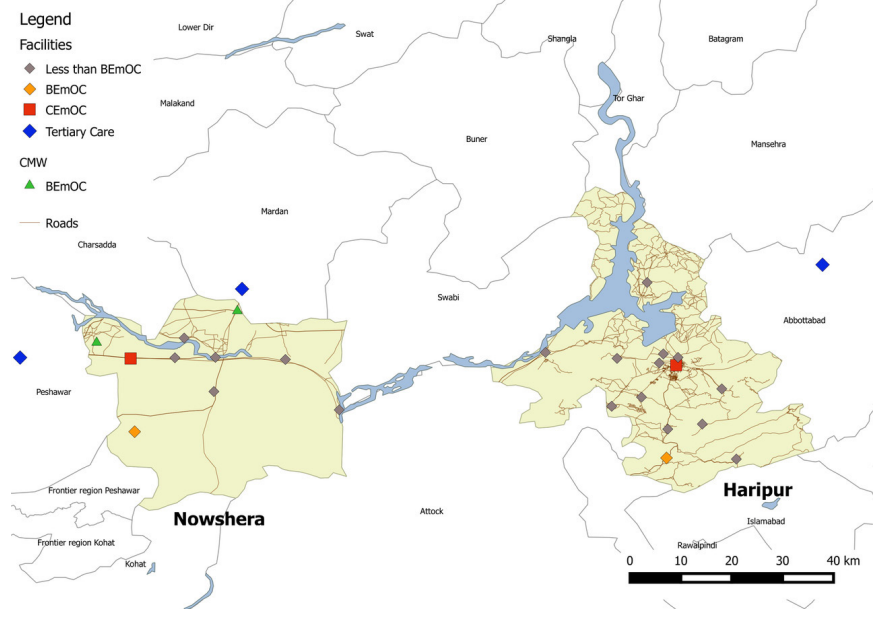

Figure 1 Spatial distribution of interviewed healthcare providers. BEmOC, basic emergency obstetric care; CEmOC, comprehensive emergency obstetric care; CMV, community midwives.

basic and specialised delivery service provision, incoming and outgoing referrals, work environment and procedures, supervision, self-assessment, record keeping and reporting, patient statistics, equipment and infrastructure assessment and drug assessment.

Medical officers and female health supervisors in health facilities were also interviewed using a structured questionnaire asking about general characteristics of the facilities such as opening hours and location, staffing, basic and specialised delivery service provision. In addition, an assessment of equipment and drug availability and the facility as well as midwifery homes infrastructure was carried out. The enumerators verified the information through observations.

Health facility interviews took place at the respective facility face-to-face with staff directly engaged in delivery services. Interviews with the CMWs took place at midwifery homes. The health facility interviews have been conducted paper-based while interviews with CMWs were entered directly into tablets using Open Data Kit (ODK) software.

\section{Patient and public involvement}

Patients were not involved in the design of the study and no direct interactions with patients were studied. Results were disseminated to health facilities and public health officials of the region through a written report and a series of workshop presentations.

\section{Analysis}

The data from the survey asking about seven key interventions, namely the administration of parenteral antibiotics, uterotonic drugs, parenteral anticonvulsants, the manual removal of placenta, the removal of retained products of conception, assisted vaginal delivery and neonatal resuscitation conducted in the last 3 months prior to the interview, were analysed descriptively. Following the UN handbook on monitoring EmOC, health facilities and CMWs have been classified as a BEmOC or CEmOC health provider accordingly. ${ }^{10}$ STATA V.14 ${ }^{25}$ was used to analyse the data.

\section{RESULTS Number of births}

The total number of births recorded in public health facilities is approximately 14429 between January and December 2015 with 7260 and 7169 births taking place in Haripur and Nowshera, respectively. Of births taking place in public health facilities, the majority, about $80 \%$ in Haripur and $50 \%$ in Nowshera, were performed at the corresponding $\mathrm{DHQH}$. The remaining births in Haripur were primarily conducted in BHUs, while about $40 \%$ of the total births in Nowshera were conducted in civil hospitals.

Between January and December 2015, there was a total of 5371 births: 1626 births at the 32 CMWs in Haripur and 3745 births at the 53 CMWs in Nowshera. This gives a yearly average of 51 and 71 births per CMW in Haripur and Nowshera, respectively, with rather large variation within the districts.

Taking the sum of total births by health facilities and CMWs results in 19800 births conducted by public health cadres. Based on the estimations of total live births in both districts, those births account for roughly $30 \%$ of all births in the Districts Haripur and Nowshera.

\section{Provision of emergency obstetric care} Health facilities

As depicted in table 1 , the assessment of the provision of full BEmOC services shows that only two facilities in each district qualify as BEmOC facilities. In the last 3 months before assessment, the $\mathrm{DHQH}$ and one hospital in Haripur, report performing basic emergency obstetric care services. In Nowshera, one RHC and one hospital provided all BEmOC services. It is remarkable that the DHQH in Nowshera does not qualify as BEmOC facility as it reported to not have administered oxytocin for the treatment of postpartum haemorrhage. One out of five RHC administered magnesium sulfate in the previous 3 months and one RHC in Nowshera was able to provide all seven interventions necessary to be classified as a BEmOC facility. A minority of the health providers support assisted vaginal delivery, neonatal resuscitation and the administration of magnesium sulfate.

In order to qualify as a CEmOC facility, health facilities need to provide caesarean sections and blood transfusions, in addition to the seven BEmOC service functions. In Haripur, the $\mathrm{DHQH}$ is the only facility providing caesareans and blood transfusion. In Nowshera, two public health providers, one hospital and the DHQH report to maintain these additional comprehensive services. Overall, as the DHQH in Nowshera does not qualify as a BEmOC facility, it consequentially does not qualify as a CEmOC facility. Given these results, there is 
Table 1 BEmOC signal functions of public health facilities

\begin{tabular}{|c|c|c|c|c|c|c|c|c|}
\hline & \multicolumn{5}{|c|}{ Haripur } & \multicolumn{3}{|c|}{ Nowshera } \\
\hline & $\begin{array}{l}\mathrm{BHU} \\
(\mathrm{n}=7)\end{array}$ & $\begin{array}{l}\mathrm{MCHC} \\
(\mathrm{n}=1)\end{array}$ & $\begin{array}{l}\mathrm{RHC} \\
(\mathrm{n}=2)\end{array}$ & $\begin{array}{l}\text { Hospital } \\
(n=3)\end{array}$ & $\begin{array}{l}\text { DHQH } \\
(n=1)\end{array}$ & $\begin{array}{l}\mathrm{RHC} \\
(\mathrm{n}=5)\end{array}$ & $\begin{array}{l}\text { Hospital } \\
(n=2)\end{array}$ & $\begin{array}{l}\mathrm{DHQH} \\
(\mathrm{n}=1)\end{array}$ \\
\hline Parenteral administration of antibiotics & 5 & 1 & 2 & 3 & 1 & 5 & 2 & 1 \\
\hline Parenteral administration of oxytocin & 7 & 1 & 2 & 2 & 1 & 5 & 2 & 0 \\
\hline $\begin{array}{l}\text { Parenteral administration of magnesium } \\
\text { sulfate }\end{array}$ & 4 & 0 & 1 & 1 & 1 & 1 & 1 & 1 \\
\hline Assisted vaginal delivery & 1 & 0 & 0 & 2 & 1 & 3 & 2 & 1 \\
\hline Manual removal of placenta & 6 & 0 & 2 & 2 & 1 & 5 & 2 & 1 \\
\hline Removal of retained products of conception & 7 & 0 & 1 & 2 & 1 & 5 & 1 & 1 \\
\hline Neonatal resuscitation with bag and mask & 4 & 1 & 1 & 2 & 1 & 3 & 2 & 1 \\
\hline BEmOC facility & 0 & 0 & 0 & 1 & 1 & 1 & 1 & 0 \\
\hline Caesarean section & 0 & 0 & 0 & 0 & 1 & 0 & 1 & 1 \\
\hline Blood transfusions & 0 & 0 & 0 & 0 & 1 & 0 & 1 & 1 \\
\hline CEmOC facility & 0 & 0 & 0 & 0 & 1 & 0 & 1 & 0 \\
\hline
\end{tabular}

BEmOC, basic emergency obstetric care; BHU, basic health units; CEmOC, comprehensive emergency obstetric care; DHQH, District Headquarter Hospitals; MCHC, maternal and child health centre; RHC, rural health centre.

one CEmOC facility available in each district with one additional facility classified as a BEmOC facility.

\section{Community midwives}

Prior to the survey, only two CMWs from Nowshera out of 85 performed all seven BEmOC services. The frequency of different BEmOC services, carried out by the CMWs varies quite substantially (table 2 ). In both districts, over $80 \%$ of the CMWs provide parenteral administration of oxytocin and neonatal resuscitation. Manual removal of the placenta is provided by a larger percentage of CMWs in Nowshera (91\%), while only $69 \%$ of CMWs in Haripur can perform it. Parenteral administration of antibiotics has been provided in both districts by $>60 \%$ of the CMWs. Removal of retained products of conception, assisted vaginal delivery and parenteral administration of magnesium sulfate has been provided in both districts by $<40 \%$ of the CMWs.

\section{Proportion of births in EmOC public sector facilities}

The UN minimum recommendations propose that at least $15 \%$ of all births should occur in health facility settings, based on the assumption that about $15 \%$ of women face complications during delivery. ${ }^{10}$ For Haripur, 5740 (20\%) births took place in EmOC facilities, mainly the DHQH. In Nowshera, the proportion of births that occurred in public EmOC health facilities equates to only 8\% (2815 births) of the estimated 36200 live births in this district, well below the UN benchmark.

\section{Availability of EmOC}

The minimal acceptable level of EmOC availability is defined as at least four BEmOC and one CEmOC health facility, serving a population of $500000 .{ }^{10}$ For Haripur and Nowshera, these recommendations would result in a minimum number of 10 and $12 \mathrm{EmOC}$ health facilities

Table 2 Basic emergency obstetric care (BEmOC) provision by community midwives (CMWs)

\begin{tabular}{|c|c|c|c|c|}
\hline \multirow[b]{2}{*}{ BEmOC services } & \multicolumn{2}{|c|}{$\begin{array}{l}\text { Haripur } \\
(\mathrm{n}=32)\end{array}$} & \multicolumn{2}{|c|}{$\begin{array}{l}\text { Nowshera } \\
(n=53)\end{array}$} \\
\hline & $\mathbf{n}$ & $\%$ & $\mathbf{n}$ & $\%$ \\
\hline Parenteral administration of antibiotics & 22 & 69 & 39 & 74 \\
\hline Parenteral administration of oxytocin & 29 & 91 & 45 & 85 \\
\hline Parenteral administration of magnesium sulfate & 8 & 25 & 12 & 23 \\
\hline Assisted vaginal delivery & 11 & 34 & 28 & 53 \\
\hline Manual removal of placenta & 22 & 69 & 48 & 91 \\
\hline Removal of retained products of conception & 12 & 38 & 21 & 40 \\
\hline Neonatal resuscitation & 28 & 88 & 43 & 81 \\
\hline Number of CMWs providing all BEmOC services & 0 & 0 & 2 & 4 \\
\hline
\end{tabular}


Table 3 Shortage of BEmOC and CEmOC facilities

\begin{tabular}{|c|c|c|c|c|c|c|}
\hline \multirow[b]{2}{*}{ Classification } & \multicolumn{3}{|l|}{ Haripur } & \multicolumn{3}{|l|}{ Nowshera } \\
\hline & $\begin{array}{l}\text { Assessed } \\
\text { availability }\end{array}$ & $\begin{array}{l}\text { WHO } \\
\text { recommendation }\end{array}$ & Shortage & $\begin{array}{l}\text { Assessed } \\
\text { availability }\end{array}$ & $\begin{array}{l}\text { WHO } \\
\text { recommendation }\end{array}$ & Shortage \\
\hline CEmOC & 1 & 2 & 1 & 1 & 2 & 1 \\
\hline
\end{tabular}

$\mathrm{BEmOC}$, basic emergency obstetric care; $\mathrm{CEmOC}$, comprehensive emergency obstetric care.

respectively, including two CEmOC facilities in each district (table 3). Following this benchmark and our obtained results from both districts, Haripur lacks seven BEmOC facilities and one CEmOC facility and Nowshera has a shortage of nine BEmOC facilities and one CEmOC facility. Although health cadres outside of health institutions do not use these benchmarks to assess the adequacy of service provision, considering the results of the CMW assessment with only two CMWs in Nowshera providing all BEmOC services, the district of Nowshera still lacks seven BEmOC health providers. Taking the data from Nowshera and Haripur together shows that both districts have only $11 \%$ (2 out of 18 recommended) BEmOC and $50 \%$ (2 out of 4) CEmOC facilities of the recommended minimum number of facilities available to its estimated population of about 2.3 million.

\section{Low performance of signal functions}

A minority of health facilities and CMWs conduct several signal functions. There are various reasons for these findings. Following the UN guidelines, ${ }^{10}$ signal functions need to be performed in the 3 months prior to the assessment. Despite the fact that our sample only covered health facilities with an average of at least three births per month, it can be assumed that there has not been enough cases to perform all of the signal functions. It is specified by the local health authorities that high-risk cases should be referred before complications occur, especially for primary health facilities and CMWs.

In the event of maternal and newborn complications, the large majority of CMWs, BHUs and RHCs report to refer the cases to the local DHQH and to tertiary healthcare providers in Abottabad and Peshawar.

\section{Medical equipment and drugs availability}

Another aspect concerns the availability of equipment and medication needed to support the interventions. For instance, only $23 \%$ of all CMWs and $45 \%$ of all health facilities administer magnesium sulfate (table 1 and table 2). The findings from the drug assessment revealed that only $13 \%$ of CMWs and $45 \%$ of health facilities had magnesium sulfate available on the day of the interview. Three health facilities from each district $(27 \%)$ and 35 CMWs (42\%) never had magnesium sulfate available. Of the CMWs and health facilities that generally had access to magnesium sulfate, $49 \%$ and $33 \%$ of those, reported to be out of stock in the past 3 months.
Overall, these findings highlight that in these regions, provision of the safe and affordable childbirth facilities are far from acceptable levels and even the existing services including CMWs face major difficulties as such were having adequate access to equipment and medication. These observations are not limited to KP and results from studies of CMWs conditions in other regions in Pakistan, for example, in districts of northern Punjab ${ }^{26}$ reported the same issues. It should be noted that alongside the lack of facilities, cultural and low level of literacy skills among women in poorer regions of Pakistan adds to the complexity of its case where interventions at the demand side is equally required. ${ }^{27}$

\section{DISCUSSION}

As the KP Health Sector Strategy for 2010-17 stated, one of the key challenges in this region is widespread poverty. For those in rural areas with poorer population it is even more difficult to access private facilities which adds to the burden of household out-of-pocket spending on healthcare. ${ }^{28}$ Following the introduction of $\mathrm{MNCH}$ and similar government and global initiatives, this study reviewed the status of the provision of care by CMWs and government hospitals aiming to identify any shortcomings by considering seven key medical services that form the BEmOC.

We conclude that the districts of Haripur and Nowshera in KP, Pakistan, show a significant shortage of available public BEmOC and CEmOC facilities. With only $11 \%$ of the recommended number of BEmOC facilities available in both districts, we find a strong underprovision of basic obstetric healthcare. Overall, $30 \%$ of the estimated total live births in both districts are conducted by CMWs and public health facilities. An estimated $22 \%$ of all births take place in public health facilities in both districts, of which $41 \%$ were conducted at health facilities that did not meet the BEmOC criteria. Further efforts to increase institutional births should keep the BEmOC and CEmOC capabilities of the public health facilities in mind and improve capabilities and quality along with the expansion of institutional births. We would like to note that the study findings are a snapshot representative for the period when the data were collected. Local health authorities were informed about the study findings prior to the publication and it is possible that this information has led to changes. The study documents that universal institutional births were prioritised before the available health 
facilities had sufficient BEmOC and CEmOC capabilities. The survey in this paper focuses on the provision of essential obstetric care by the public health sector. Accounting for the availability of private healthcare providers and its associated costs will help to obtain a comprehensive understanding of the accessibility of EmOC services in these districts.

Public health authorities in other LMICs are encouraged to assess BEmOC and CEmOC capabilities to complement policies that aim at increasing institutional births. For Pakistan, a meta-analysis of existing literature and evidence gathered from various provinces and districts to build a map of status of facilities at the national level could be particularly helpful in identifying areas that are most deprived as well as providing opportunities where it is possible to transfer experience and lessons learnt across various regions.

Contributors PB wrote the first draft of the paper, supported the acquisition of the data, conceptualised the study and analysed data. AH and MK supported the acquisition of the data and contributed to the conceptualisation of the study. JK supported the acquisition of the data, conceptualised the study and contributed to the interpretation of results and writing. IM contributed to the conceptualization of the study and the interpretation of results and writing. SV supervised the project, conceptualised the study and contributed to the interpretation of results and writing

Funding This study was funded by the Deutsche Gesellschaft für Internationale Zusammenarbeit (GIZ).

Disclaimer The views expressed in this manuscript are those of the authors and do not necessarily reflect those of the funder. The GIZ had no influence in the design of the study, data collection, analysis, interpretation of data, writing the manuscript or decision to publish.

Competing interests None declared.

Patient consent for publication Not required.

Ethics approval Ethical approval was granted by the National Bioethics Committee (NBC) Pakistan, Research Ethics Committee, with the ethical approval number 4-87/15/NBC-190/RDC/994.

Provenance and peer review Not commissioned; externally peer reviewed.

Data sharing statement The datasets used and analysed during the current study are available from the corresponding author on reasonable request.

Open access This is an open access article distributed in accordance with the Creative Commons Attribution Non Commercial (CC BY-NC 4.0) license, which permits others to distribute, remix, adapt, build upon this work non-commercially, and license their derivative works on different terms, provided the original work is properly cited, appropriate credit is given, any changes made indicated, and the use is non-commercial. See: http://creativecommons.org/licenses/by-nc/4.0/.

\section{REFERENCES}

1. WHO, UNICEF, UNFPA, World Bank Group and UNDP. Trends in maternal mortality: 1990 to 2015: estimates by WHO, UNICEF, UNFPA, World Bank Group and the United Nations Population Division, 2015.

2. WHO. Every newborn: An action plan to end preventable deaths. Geneva: World Health Organization, 2014.

3. UNICEF. Levels \& Trends in Child Mortality: Report 2015, 2015.

4. NIPS, ICF. Pakistan Demographic and Health Survey 2012-13. Islamabad, Pakistan, Calverton, Maryland,USA, 2013.

5. WHO. Making pregnancy safer: The critical role of the skilled attendant: a joint statement by WHO, ICM and FIGO. Geneva: World health organization $(\mathrm{WHO})$ : Department of reproductive health and research (RHR), 2004

6. WHO. WHO Recommended interventions for improving maternal and newborn health. $2009 \mathrm{http}: / / a p p s . w h o . i n t / i r i s / b i t s t r e a m / 10665 /$ 69509/1/WHO_MPS_07.05_eng.pdf?ua=1\&ua=1

7. Government of Khyber Pakhtunkhwa. Comprehensive Development Strategy 2010-2017, 2010.

8. Kruk ME, Leslie HH, Verguet S, et al. Quality of basic maternal care functions in health facilities of five African countries: an analysis of national health system surveys. Lancet Glob Health 2016;4:e845-e855.

9. Leslie $\mathrm{HH}$, Fink $\mathrm{G}$, Nsona $\mathrm{H}$, et al. Obstetric facility quality and newborn mortality in Malawi: a cross-sectional study. PLoS Med 2016;13:e1002151.

10. UN. Monitoring emergency obstetric care: a handbook. Geneva, Switzerland: World Health Organization, 2009.

11. Islam M, Yoshida S. Women are still deprived of access to lifesaving essential and emergency obstetric care. Int J Gynaecol Obstet 2009;106:120-4.

12. Paxton $A$, Maine $D$, Freedman $L$, et al. The evidence for emergency obstetric care. Int J Gynaecol Obstet 2005;88:181-93.

13. Campbell OMR, Graham WJ. Strategies for reducing maternal mortality: getting on with what works. The Lancet 2006;368:1284-99.

14. Rammohan $\mathrm{A}$, lqbal $\mathrm{K}$, Awofeso $\mathrm{N}$. Reducing neonatal mortality in India: critical role of access to emergency obstetric care. PLoS One 2013;8:e57244

15. Yakoob MY, Ali MA, Ali MU, et al. The effect of providing skilled birth attendance and emergency obstetric care in preventing stillbirths. BMC Public Health 2011;11(Suppl 3):S7.

16. Averting Maternal Death and Disability, Working Group on Indicators. Averting maternal death and disability. Program note. Using UN process indicators to assess needs in emergency obstetric services: Pakistan, Peru and Vietnam. Int J Gynaecol Obstet 2002;78:275-82.

17. Government of Pakistan. National program for maternal, newborn and child health (2006-2012): Government of Pakistan's Initiative for Millennium Developments Goals 4 and 5. Islamabad, Pakistan, 2013.

18. Mahmud G, Zaman F, Jafarey $S$, et al. Achieving millennium development goals 4 and 5 in Pakistan. BJOG 2011;118(Supp. 2):69-77.

19. Ali M, Hotta M, Kuroiwa C, et al. Emergency obstetric care in Pakistan: potential for reduced maternal mortality through improved basic EmOC facilities, services, and access. Int J Gynaecol Obstet 2005;91:105-12.

20. Utz B, Zafar S, Arshad N, et al. Status of emergency obstetric care in four districts of Punjab, Pakistan-results of a baseline assessment. $J$ Pak Med Assoc 2005;65:480-5.

21. Ansari MS, Manzoor R, Siddiqui N, et al. Access to comprehensive emergency obstetric and newborn care facilities in three rural districts of Sindh province, Pakistan. Health Res Policy Syst 2015;13:S55

22. Pakistan Bureau of Statistics. Pakistan Social And Living Standards Measurement Survey (PSLM) 2013-14 National / Provincial. http:// www.pbs.gov.pk/sites/default/files//pslm/publications/pslm2013_14/ complete_report_psIm_2013_14.pdf (Accessed 7 Feb 2019).

23. Pakistan Bureau of Statistics. Pakistan Social And Living Standards Measurement Survey (PSLM) 2014-15 National / Provincial/District. http://www.pbs.gov.pk/sites/default/files/ps/m/publications/PSLM 2014-15_National-Provincial-District_report.pdf (Accessed 7 Feb 2019).

24. UN. World population prospects: the 2015 revision, data booklet. 2015 https://esa.un.org/unpd/wpp/Publications/Files/WPP2015 DataBooklet.pdf (Accessed 24 Nov 2016).

25. StataCorp. Stata statistical software: release 14. College Station TX: StataCorp LP, 2015.

26. Sarfraz M, Hamid S. Challenges in delivery of skilled maternal care - experiences of community midwives in Pakistan. BMC Pregnancy Childbirth 2014;14:59.

27. Mumtaz Z, Salway S, Bhatti A, et al. Improving maternal health in Pakistan: toward a deeper understanding of the social determinants of poor women's access to maternal health services. Am J Public Health 2014:104(Suppl 1):S17-S24.

28. trfpakistan. Khyber pakhtunkhwa health sector strategy 2010 2017. 2010 http://www.trfpakistan.org/Portals/18/TRF\%20reports/ Strategies/KPK\%20Health\%20Sector\%20Strategy\%202010.pdf? ver=2017-03-22-181031-467 (Accessed 7 Feb 2019). 